\section{Discussion}

Although a number of centres have described their experience of cytotoxic chemotherapy in pregnancy, ${ }^{24}$ there is very little information in these reports about the haematological status of the babies. We know of only one previous case report in which the blood count changes were documented. ${ }^{5}$ (The baby had isolated neutropenia and the baby's bone marrow status was not assessed.)

In this case the baby developed pancytopenia within 30 hours, and circulating haemopoietic progenitor cell studies confirmed marrow suppression: CFU-GM were reduced at birth, consistent with the severe neutropenia. They increased to above normal levels by day 5 . While this may have been due to the administration of G-CSF, it is more likely to have been the result of a rebound phenomenon as occurs in adults after chemotherapy. BFU-e were towards the lower end of our normal range at birth but doubled in the first five days of life. This contrasts with the usual pattern in which the number of BFU-e is highest in the first 24 hours of life and falls during the following week. At birth numbers of cultured megakaryoblasts and megakaryocytes were severely reduced. We have found that this predicts the development of thrombocytopenia during the first week of life. ${ }^{6}$ All progenitor markers had returned to normal by day 33.

We believe this is the first neonatal case of its kind to be investigated by serial study of the three main types of haemopoietic progenitor. The data show that the baby's marrow was actively regenerating after an acute insult. In this instance cytotoxic chemotherapy given to the mother caused fetal marrow suppression, roughly equal to the extent seen in the mother. This may have been due to the use of etoposide in this case, a relatively new antineoplastic agent which crosses the placenta and is particularly myelosuppressive. It is also highly mutagenic, ${ }^{7}$ raising the possibility of late treatment induced leukaemia in this baby.

This case illustrates that severe fetal marrow suppression may occur if women receive full dose cytotoxic chemotherapy during pregnancy. Any decision to expedite delivery in this situation should take into account the possible haematological status of the fetus (which, if appropriate, could be assessed by cordocentesis). At birth the baby may have severe haematological abnormalities, require immediate resuscitation, and ongoing neonatal intensive care. After the neonatal period repeated follow up of the baby is essential, in view of the possible long term effects of cytotoxic agents.

All obstetricians, haematologists, and neonatal paediatricians involved in the care of such pregnant women should be aware of the potential risks to the fetus, ensure that delivery and neonatal care take place in an appropriate setting, and subsequently plan careful long term follow up of the baby.

We gratefully acknowledge the support of Action Research in the development of the megakaryocyte progenitor assay systems the development of the $m$
used in this investigation.

We also acknowledge the help of Dr Ruth Warwick, consulWe also acknowledge the help of Dr Ruth Warwick, consul-
tant haematologist, National Blood Transfusion Service, tant haematologist, National Blood Transfusion Service, haematologist, Hillingdon Hospital, Uxbridge, Middlesex.

1 Slade R, James DK. Pregnancy and maternal malignant haematological disorders. In: Turner TL, ed. Perinatal haematological problems. Chichester: John Wiley \& Sons Ltd, 1991: 23-38.

2 Aviles A, Dias-Maqueo JC, Talavera A, Guzman R, Garcia EL. Growth and development of children of mothers treated with chemotherapy during pregnancy: Current treated with chemotherapy during pregnancy: Curren
status of 43 children. Am $\mathscr{f}$ Hematol 1991; 36: 243-8.

status of 43 children. Am f Hematol 1991; 36: 243-8.
3 Urabe A. Murphy MJ, Haghbin M, Gee TS. Erythroid rabe A. Murphy MJ, Haghbin M, Gee TS. Erythroid
progenitors (BFU-e and CFU-e) in acute leukemia. $\mathcal{f}$ Clin Pathol 1979; 32: 666-9.

4 Roy V, Gutteridge CN, Nysenbaum A, Newland AC. Combination chemotherapy with conservative obstetric management in the treatment of pregnant patients with acute myeloblastic leukemeatment of pregnant patients with acute myeloblastic leukemia. Clin Lab Haematol 1989; 11: 171-8. effects of maternal cancer chemotherapy. Case report. Brf $\mathcal{F}$
Obstet Gynaecol 1989; 96: 1099-100.

6 Anonymous. Abstract. Early Hum Devel 1994; 36: 228.

7 Pedersen-Bjergaard J, Daugaard G, Hansen SW, Philip P Pedersen-Bjergaard J, Daugaard G, Hansen SW, Philip P,
Larsen SO, Rørth M. Increased risk of myelodysplasia and leukemia after etoposide, cisplatin, and bleomycin for germ-cell tumours. Lancet 1991; 338: 359-63.

\title{
Poor prognosis after prolonged ventilation for bronchopulmonary dysplasia
}

\author{
Mary Wheater, Janet M Rennie
}

\begin{abstract}
Of 47 babies mechanically ventilated for more than 27 days for bronchopulmonary dysplasia 20 eventually died. There were no neurodevelopmentally intact survivors among those who were ventilated for more than $\mathbf{5 0}$ days. Days of ventilation more powerfully predicted outcome than ultrasound evidence of brain injury.

(Arch Dis Child 1994; 71: F210-F211)
\end{abstract}

Prolonged neonatal mechanical ventilation is an indicator of poor prognosis, but there are
Correspondence to: Dr Rennie.

Accepted 19 July 1994 few estimates of the likelihood of normal survival. Overstreet et al studied 144 infants requiring more than 30 days' mechanical ventilation. ${ }^{1}$ Survival to discharge was related to duration of ventilation, with $90 \%$ mortality for babies ventilated for more than 120 days. Long term survival and neurodevelopmental outcome were not reported.

Ventilation for more than 21 days was a powerful predictor of developmental delay at 18 months in infants with birth weights below 1201 g. $^{2}$ Luchi et al detected no relationship between duration of mechanical ventilation and neurodevelopmental outcome among 27 
survivors of birth weight $540-1690 \mathrm{~g}$ ventilated for up to 128 days. ${ }^{3}$ We investigated mortality and neurodevelopmental morbidity among infants admitted to the Cambridge neonatal intensive care unit between 1985 and 1992 and ventilated for 28 days or more.

\section{Methods}

We identified all babies fulfilling our entry criteria from a purpose written prospectively maintained computerised database. Extensive antenatal, perinatal, and neonatal data were available. Brain injury was diagnosed by weekly cranial ultrasound and defined as including intraventricular haemorrhage, intracerebral haemorrhage, and periventricular leukomalacia but not subependymal haemorrhage alone. Prolonged ventilation was defined as artificial ventilation for more than 27 days because of bronchopulmonary dysplasia (BPD), which was diagnosed on the basis of changes on $x$ ray film and a requirement for supplementary oxygen at 28 days in babies initially ventilated for respiratory distress syndrome. Babies ventilated for more than 27 days because of neurological disease or pulmonary hypoplasia were excluded.

Infants born after $1987(n=16)$ were seen at 9 and 18 months after term and assessed using the neurological examination described by Amiel-Tison and Stewart ${ }^{4}$ and the Bayley scales of infant development. ${ }^{5}$ Information regarding the other survivors was obtained from hospital records, child development clinics, or general practitioners. Disability was defined as significant developmental delay (developmental scores, when available, of more than two standard deviations below the mean), blindness, or cerebral palsy. Statistical analysis was performed using the Statistical Package for the Social Sciences.

\section{Results}

During the study period 798 very low birth weight (VLBW) babies were admitted, of whom 645 required mechanical ventilation and 164 developed BPD. Forty five VLBW babies (28 boys) were ventilated for 28 days or more. Only two babies of birth weight of more than $1500 \mathrm{~g}$ required ventilation for 28 days or more. The median birth weight of the whole

Table 1 Number of children normal, disabled, or dead at 18 months according to cerebral ultrasound appearances

\begin{tabular}{lllr}
\hline Ultrasound scan & Normal & Disabled & Dead \\
\hline Normal or subependymal & & & \\
$\quad$ haemorrhage & 7 & 8 & 11 \\
Intraventricular haemorrhage & 0 & 3 & 5 \\
Parenchymal haemorrhage & 1 & 4 & 2 \\
Any periventricular leukomalacia & 2 & 2 & 2 \\
Total with brain injury & 3 & 9 & 9 \\
\hline
\end{tabular}

Table 2 Number of children normal, disabled, or dead at 18 months according to duration of mechanical ventilation

\begin{tabular}{lllc}
\hline Days ventilated & Normal & Disabled & Dead \\
\hline $28-40$ & 8 & 11 & 5 \\
$41-50$ & 2 & 4 & 5 \\
$>50$ & 0 & 2 & 10 \\
\hline
\end{tabular}

group of 47 was $806 \mathrm{~g}$ (range 438-1595) and the median gestational age was 26 weeks (range 23-31). The median duration of ventilation was 40 days (maximum 100). Twenty babies died at a median postnatal age of 183 days (range 34-604 days), three never having been off a ventilator. In two of these cases death was the result of a decision to withdraw ventilatory support. Ten died after discharge from our neonatal unit, but only four had ever been home. Three of these died aged more than 1 year but all died of respiratory disease. There was ultrasound evidence of brain injury in nine subjects who died and 12 survivors (table 1 ).

Of the 27 surviving children only 10 were not disabled; all were ventilated for 50 days or less (table 2). The predominant disability was cerebral palsy ( 15 children). Two were cognitively impaired without major motor disability but one of these was also blind. Three of the 10 normal survivors had ultrasound evidence of brain injury as defined before, compared with $9 / 17(52 \%)$ of surviving handicapped children (table 1). Regression analysis showed that an adverse outcome (death or handicap) was significantly associated with days of ventilation $(p=0.0017)$ but not with the presence of brain injury on ultrasound scan, sex, birth weight, or gestational age.

\section{Discussion}

In Cambridge, tracing of all babies who received prolonged ventilation revealed the full extent of the high mortality $(43 \%)$ and morbidity. The findings reinforce the importance of obtaining nearly complete follow up, a point first raised by Wariyar and Richmond. ${ }^{6}$

Our morbidity data support the findings of Bozynski et al, ${ }^{2}$ that duration of ventilation is a more powerful predictor of handicap than ultrasound evidence of brain injury. Metaanalysis of published data ${ }^{7}$ shows that for VLBW infants discharged with a normal neonatal cerebral ultrasound appearance, the risk of disability is $6 \%$ (95\% confidence interval 5 to 8 ). Our data suggest that for infants surviving prolonged ventilation this prediction should be qualified. The poor outlook after ventilation for over 40 days may be useful information for clinicians facing difficult decisions during a deterioration in the condition of these babies.

We would like to thank $\mathrm{Dr}$ Ruth Morley (MRC clinical scientist) who performed the follow up examinations. Dr Colin Morley and Dr Gill Gandy helped to maintain the database.

1 Overstreet DW, Jackson JC, van Belle G, Truog WE. Estimation of mortality risk in chronically ventilated infants with bronchopulmonary dysplasia. Pediatrics 1991; 88: 1153-60.

2 Bozynski MEA, Nelson MN, Matalon TAS, et al. Prolonged mechanical ventilation and intracranial haemorrhage: impact on developmental progress through 18 months in infants weighing 1200 grams or less at birth. Pediatrics 1987; 79: 670-6.

3 Luchi JM, Bennett FC, Jackson JC. Predictors of neurodevelopmental outcome following bronchopulmonary dysplasia. Am f Dis Child 1991; 145: 813-7.

4 Amiel-Tison C, Stewart A. Follow up studies during the first five years of life: a pervasive assessment of neurological five years of life: a pervasive assessment of
function. Arch Dis Child 1989; 64: 496-502.

5 Bayley N. The Bayley scales of infant development. New York: The Psychological Corporation, 1969.
.

6 Wariyar U, Richmond S. Morbidity and preterm delivery: importance of $100 \%$ follow up. Lancet 1989; i: 387-8.

7 Rennie JM. The newborn. In: Forfar and Armeil's textbook of paediatrics. 4th Edn. Edinburgh: Churchill Livingstone,
1992: 187-94. 